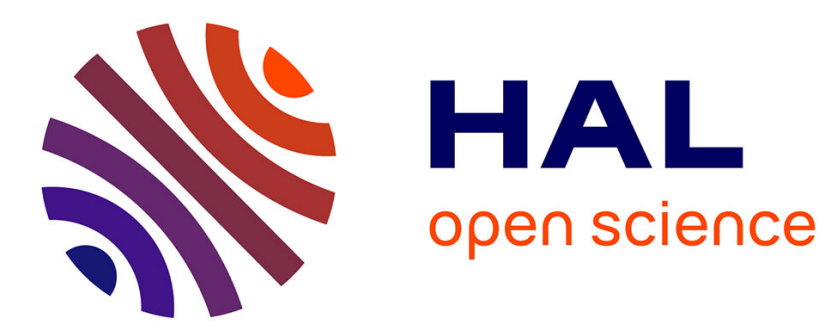

\title{
LIGHT SCATTERING FOR STRUCTURAL INVESTIGATIONS OF SILICA AEROGELS AND ALCOGELS
}

\author{
A. Beck, O. Gelsen, P. Wang, J. Fricke
}

\section{- To cite this version:}

A. Beck, O. Gelsen, P. Wang, J. Fricke. LIGHT SCATTERING FOR STRUCTURAL INVESTIGATIONS OF SILICA AEROGELS AND ALCOGELS. Journal de Physique Colloques, 1989, 50 (C4), pp.C4-203-C4-208. 10.1051/jphyscol:1989433 · jpa-00229509

\section{HAL Id: jpa-00229509 https://hal.science/jpa-00229509}

Submitted on 1 Jan 1989

HAL is a multi-disciplinary open access archive for the deposit and dissemination of scientific research documents, whether they are published or not. The documents may come from teaching and research institutions in France or abroad, or from public or private research centers.
L'archive ouverte pluridisciplinaire HAL, est destinée au dépôt et à la diffusion de documents scientifiques de niveau recherche, publiés ou non, émanant des établissements d'enseignement et de recherche français ou étrangers, des laboratoires publics ou privés. 
REVUE DE PHYSIQUE APPLIQUEE

Colloque C4, Supplément au $n^{\circ} 4$, Tome 24, avril 1989

C4-203

\title{
LIGHT SCATTERING FOR STRUCTURAL INVESTIGATIONS OF SILICA AEROGELS AND AL,COGELS
}

\author{
A. BECK, O. GELSEN, P. WANG and J. FRICKE \\ Physikalisches Institut der Universität, Am Hubland, D-8700 Würzburg, \\ F.R.G.
}

\begin{abstract}
Résumé : On décrit un appareil de diffusion qui permet l'étude des gels et des aérogels dans la lumière visible. Les formules permettant de corriger les résultats pour divers types de géométries sont explicitées. Nous reportons les résultats de diffusion obtenus pour des aérogels de silice ayant des densités de 71,105 et $143 \mathrm{Kg} / \mathrm{m}^{3}$; les longueurs de corrélation ac varient entre 10 et $25 \mathrm{~nm}$; ces valeurs diminuent lorsque la densité augmente. Nous reportons aussi les résultats de diffusion de lumière réalisés lors de la transformation sol-gel.
\end{abstract}

Abstract - A scattering apparatus is described which allows the investigation of gels and aerogels with visible light. The correction formulas for various typical geometries are derived. Scattering data for silica aerogels with densities 71,105 and $143 \mathrm{~kg} / \mathrm{m}^{-3}$ are provided; the derived correlation lenghts $a_{c}$ are in the range 10 to $25 \mathrm{~nm}$; they show a systematic decrease with increasing density. We also provide results for light scattering in a sol-gel process.

\section{1 - INTRODUCTION}

Silica aerogels /1,2/ have a wide pore size distribution, with micro, meso and macro porosity. Structural entities below or within the $10 \mathrm{~nm}$ range are conveniently investigated by small angle X-ray scattering (SAXS) /3/ and neutron scattering (SANS) /4/. Analysis of macroporosity requires values for the momentum transfer in the range $Q \leq 10^{-2} \AA^{-1}$ and thus very high angular resolution with SAXS and SANS. Small momentum transfer also occurs in light scattering experiments /5/ with access to the range $3 \cdot 10^{-4} \AA^{-1} \leq Q \leq 2 \cdot 10^{-3} \AA^{-1}$ for photons with wavelengths $\lambda$ of $633 \mathrm{~nm}$ and scattering into angles $20^{\circ} \leq \theta \leq 160^{\circ}$. A discussion of structural investigations of aerogels has been presented recent $1 \mathrm{y} / 6 /$.

\section{2 - THEORY}

An exponential correlation function $\gamma(r)=\exp \left(-r / a_{c}\right)$ is assumed to describe the structural fluctuations in density; $r$ is the length of the "measuring stick" and $a_{c}$ the correlation length. This equation can be derived for a two phase medium $/ 7 /$. The scattered intensity from such a medium is given by

$$
I(\theta) \sim \frac{1}{\left(1+\left(Q \cdot a_{c}\right)^{2}\right)^{2}}
$$

where $Q=2(2 \pi / \lambda) \cdot \sin (\theta / 2)$ is the momentum transfer divided by $h$; $\theta$ is the scattering-angle, $\lambda$ the wavelength within the specimen. If only small deviations from isotropic or Rayleigh scattering is observed, the measured intensities are usually plotted as $\mathrm{I}^{-1 / 2}$ versus $\sin ^{2}(\theta / 2)$ or -- for smal1, correlation lengths $\left(a_{c} \cdot Q \ll \cdot 1\right)$-- as intensity $I$ versus $\sin ^{2}(\theta / 2)$. This is known as Rayleigh-Gans approximation for which $4 \pi \cdot(n-1) \cdot a_{c}$ « holds. $n$ means the relative index of refraction. From $a_{c}$ a relation between the averaged spacial extension, $a_{c} d$ and $a_{c 2}$, of the structural entities for the two phases can be derived:

$$
1 / a_{c}=1 / a_{c 1}+1 / a_{c 2} \text {. }
$$

For the two quantities $a_{c 1}=a_{c} / \emptyset_{2}$ and $a_{c 2}=a_{c} / \emptyset_{1}$ holds. $\emptyset_{1}$ and $\emptyset_{2}$ are the volume fractions for both phases $\left(\emptyset_{1}+\phi_{2}=1\right)$.

\section{3 - EXPERTMENTAL ARRANGEMENT}

The vertically polarized He-Ne laser beam $(\lambda=633 \mathrm{~nm})$ is chopped before entering the specimen (see fig, 1). The scattered light is monitored via a photodiode system, which accepts radiation 


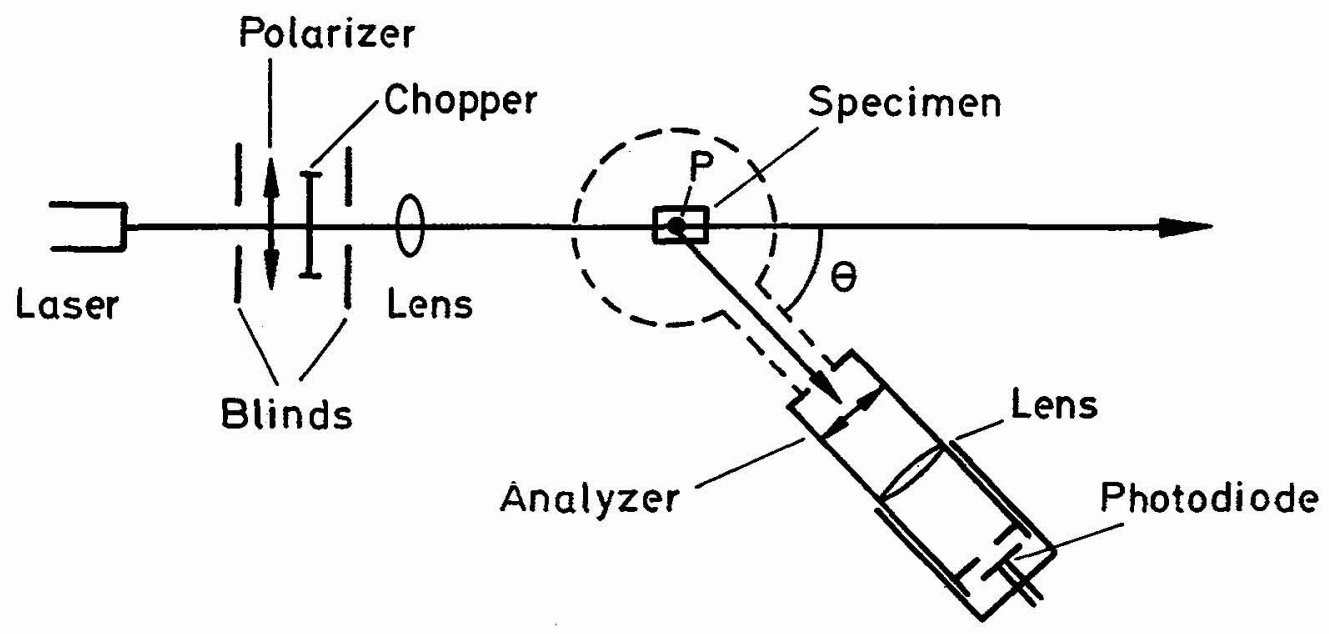

Fig. 1 - Experimental arrangement with specimen, laser beam and detector system.

within an angle of about $2^{\circ}$ and rotates about the fixed point $P$. The angular position of the detector controlled by computer can be varied from $\theta=170^{\circ}$ through $\theta=0^{\circ}$ towards negative angles in steps of $0.5^{\circ}$. The electronic signal from the scattered and transmitted intensity, respectively, is fed into a lock-in amplifier.

4 - CORRECTIONS FOR THE DETECTED SCATTERED INTENSITY

\section{1 - TILE GEOMETRY}

The scattered intensity from specimens with tile geometry has to be corrected for a series of effects (see fig. 2):

- the laser beam is attenuated due to the extinction coefficient $E$ on its way through the aerogel tile;

- the laser beam has a width 2w; thus photons being emitted from the front parts of the scattering volume have a shorter path length within the specimen.

- the scattered radiation has to pass through parts of the tile before reaching its surface (and then the detector); this causes an attenuation which is strongly dependent on the scattering angle $\theta$;

- the length (generally the shape) of the viewed scattering volume changes dramatically with $\theta$.

- due to the refraction at the aerogel-air-interface the position $P^{\prime}$ of the actual scattering volume is changed (for $0^{\circ}<\theta^{\circ}<90^{\circ}$ beam-downward and for $90^{\circ}<\theta^{\circ}<180^{\circ}$ beam-upward); apparent1y the scattered intensity is emitted from the fixed point $P$.

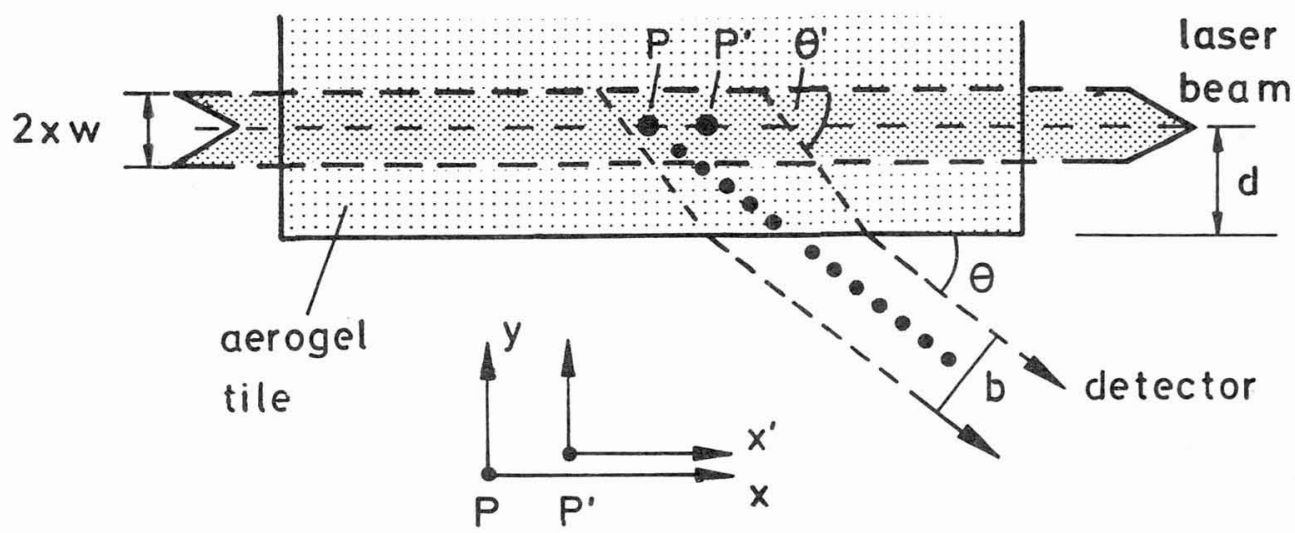

Fig. 2 - Scattering geometry within an aerogel tile. The scattering area is determined by the cverlap between the laser beam and the viewing range of the detector. The shape of the scattering area dependents strongly on the scattering angle $\theta$ (or $\left.\theta^{\prime}\right)$.

The angular dependence of the detected signal $f(\theta)$ for an isotropically scattering specimen 
is derived from sumation of the scattered intensities over the illuminated viewing range:

$$
f(\theta)=e^{-E d / \sin \theta} \int_{y=-w / 2}^{y=w / 2} e^{E y / \sin \theta} \int_{x_{\min }^{\prime}}^{x_{\max }^{\max }} e^{-E x} d x d y,
$$

with $x=x^{\top}+d \cdot H(\theta)$,

$H(\theta)=1 / \tan \theta-\cos \theta /\left(n^{2}-\cos ^{2} \theta\right)^{1 / 2}$,

$\mathrm{x}_{\mathrm{m} \pm \mathrm{n}}=-\mathrm{b} /(2 \cdot \sin \theta)+\mathrm{y} / \tan \theta$ and $\mathrm{x}_{\max }=\mathrm{b} /(2 \cdot \sin \theta)+\mathrm{y} / \tan \theta$.

After integration and for $\mathrm{Eb} /(2 \cdot \sin \theta) \ll 1$ and $E w g(\theta) \ll 1$ we get

$$
\begin{aligned}
& \frac{f\left(90^{\circ}\right)}{f(\theta)}=\frac{D\left(\left|90^{\circ}-\theta\right|\right)}{D\left(0^{\circ}\right)} \cdot e^{-E d(1-1 / \sin \theta)} \sin \theta \cdot \\
& \text { where } D(\theta)=\frac{4 \cdot K(\theta)}{n \cdot[K(\theta) / n+1]^{2}} \text { and } \\
& K(\theta)=\frac{\left[n^{2}-\sin ^{2} \theta\right]^{1 / 2}}{n \cdot \cos \theta} .
\end{aligned}
$$

The inverse of this ratio is shown in fig. 3 for typical extinction values of highly transparent aerogel tiles $\left(E=10 \mathrm{~m}^{-1}\right)$. Due to the factor $1 / \sin \theta$, one observes a dramatic

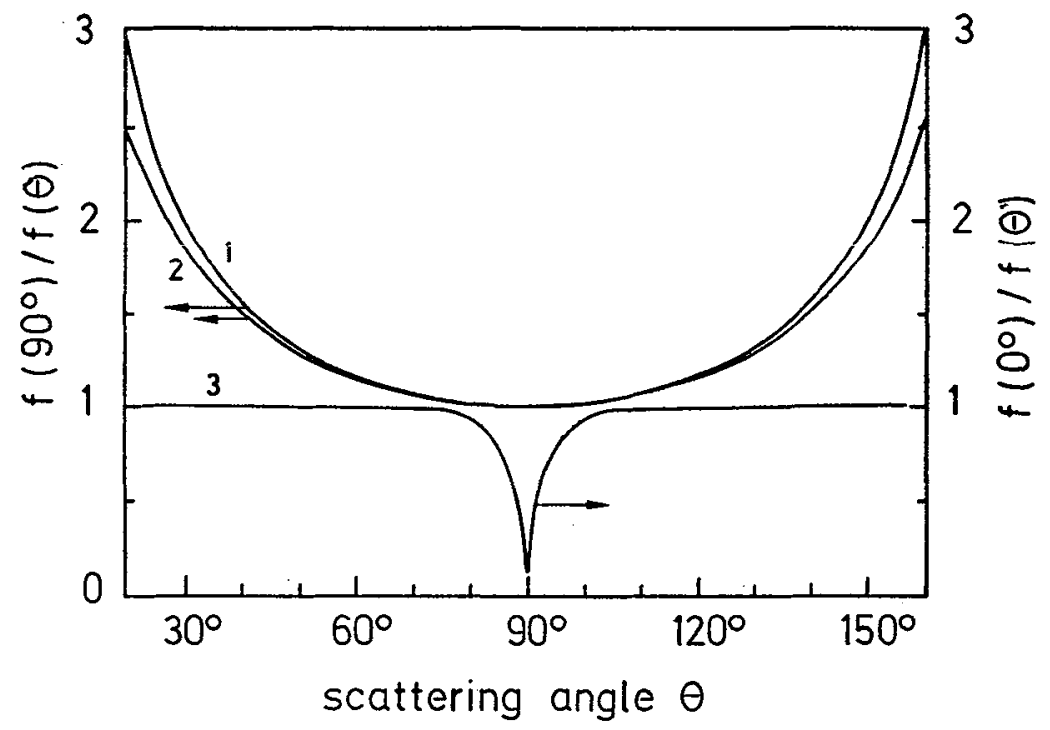

Fig. 3 - Correction functions for cylinder geometry (1) and tile geometry (2) (1eft ordinate) as well as for platelets (3) (right ordinate), for bulk extinction coefficient $E=10 \mathrm{~m}^{-1}, \mathrm{~d}=0.002 \mathrm{~m}, \mathrm{~b}=0.008 \mathrm{~m}, \mathrm{w}=0.0005 \mathrm{~m}$ and $\mathrm{n}=1.022$.

increase of $f(\theta) / f\left(90^{\circ}\right)$ for angles $60^{\circ} \geq \theta \geqslant 120^{\circ}$. In addition, $f(\theta)$ is nonsymetrical with respect to $\theta=90^{\circ}$. The transformation $\theta \rightarrow \theta^{\prime}$ (see fig. 2) is determined from the index of refraction $n$ of the aerogel tile:

$$
\frac{\sin \left(90-\theta^{\prime}\right)}{\sin (90-\theta)}=n
$$

It should be pointed perpendicular to the out that the polarization of the scattered light always has to be scattering patterns.

\section{2 - CYLINDER GEOMETRY}

In this case the correction procedure of the detected light signal is simpler (see fig. $4 \mathrm{a}$ ). 
For $w \ll r$ the remaining corrections are given as follows

$$
\frac{f\left(90^{\circ}\right)}{f(\theta)}=e^{-E b / 2 \cdot(1-1 / \sin \theta)} \cdot \frac{1-e^{-E b / \sin \theta}}{1-e^{-E b}} \cdot\left\{1-\frac{8 \cdot E \omega}{3 \pi} \cdot \frac{\sin \theta+\cos \theta-1}{\sin \theta}\right\} .
$$

This equation is depicted in $\mathrm{fig}$. 3. As can be seen the differences between the tile and the cylinder geometry become noticeable only for $\theta<60^{\circ}$ and $\theta>120^{\circ}$.

\section{3 - PLATELETS}

In this case (see fig. $4 \mathrm{~b}$ ) the width $\mathrm{b}$ for the detected radiation was always chosen large

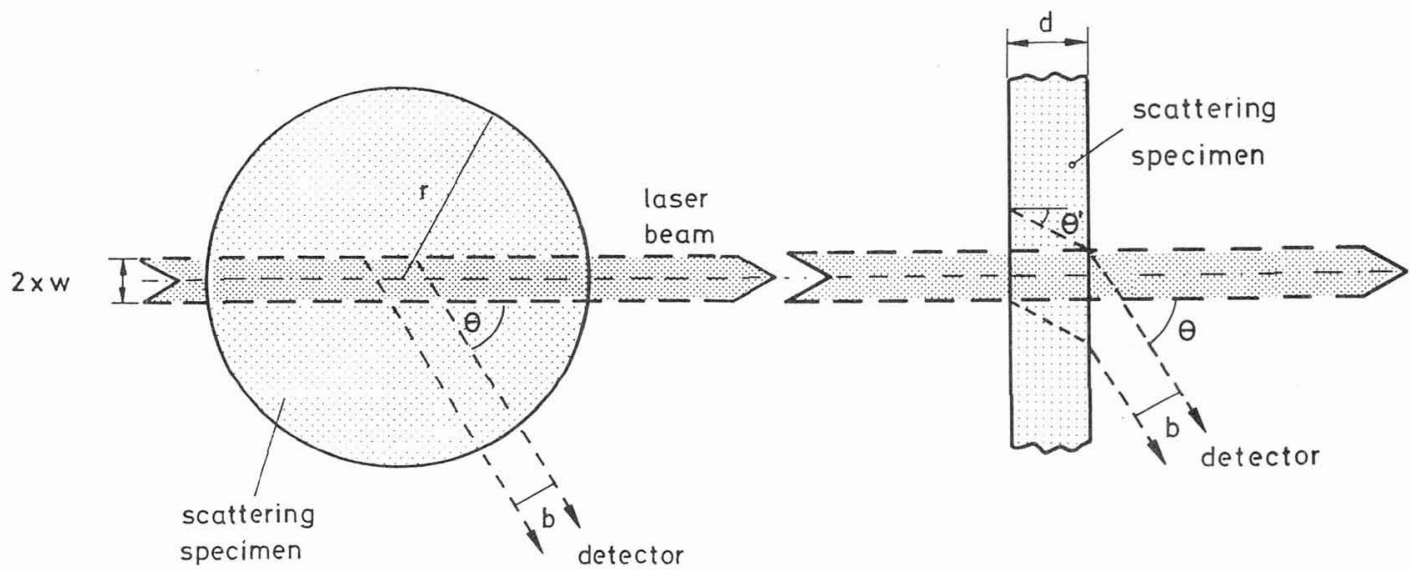

Fig. $4 a$ - Cylinder scattering geometry;

Fig. $4 b$ - Platelet scattering geonetry;

enough in order to view the total scattering volume. If the surface is smooth and does not contribute to scattering processes, the correction function becomes

$$
\frac{f\left(0^{\circ}\right)}{f(\theta)}=\frac{D(\theta)}{D\left(0^{\circ}\right)} \cdot \cos \theta \cdot \frac{1-e^{-E d(1-1 / \cos \theta)}}{E d \cdot(1-\cos \theta)} .
$$

This correction is shown in fig. 3. Critical are the scattering angles between $80^{\circ}<\theta<120^{\circ}$ upon approach of $\theta^{\prime} \approx \theta^{\prime} x_{-1 t}$, with $\theta^{\prime}$ crtt. being the angle of total reflection within the specimen.

In principle all 3 techniques are equivalent and ought to give the same scattering distribution. However, the platelet geometry is the most critical one, if bulk effects are to be studied. In this case the laser beam penetrates the surface section, which is within the detector viewing range, and thus surface scattering will mask the weak volume scattering contribution. This holds especially upon approach of the angle of total reflection.

\section{5 - MEASUREMENTS AND DISCUSSION}

Supercritical drying $/ 8 /$ of alcogels allows the production of extremely porous solid bodies of $\mathrm{SiO}_{2}$ (porosity up to 98\%) /9/. The high-quality transparent aerogel specimens produced at DESY (Hamburg) and Lund (Sweden) from TMOS (Tetramethoxysilan) under basic conditions are mainly Rayleigh scatterers and therefore show a more or less isotropic scattering pattern. The observed slopes (see fig. 5) in a $\mathrm{I}^{-1 / 2}$ versus $\sin ^{2} \theta / 2$ plot derived from a tile geometry thus are generally very small. Under close observation, however, we observe a systematic decrease 


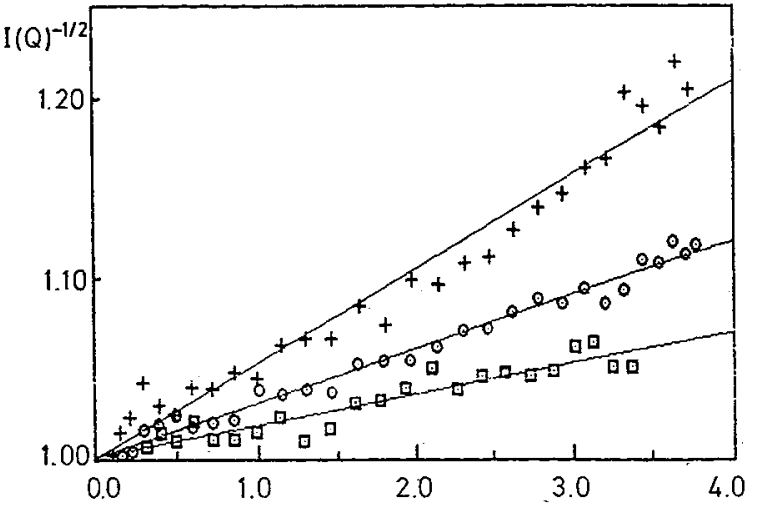

$\left(Q / 10^{-3} \AA^{-1}\right)^{2}$

Fig. 5 - Scattering behavior of monolithic aerogels produced under basic conditions; the data sets belong to the densities $\rho \approx 71 \mathrm{~kg} / \mathrm{m}^{3}(+) \rho \approx 105 \mathrm{~kg} / \mathrm{m}^{3}(0)$ and $\rho=143 \mathrm{~kg} / \mathrm{m}^{3}$ (פ); the corresponding correlation lengths are $a_{c}=23 \mathrm{~nm}, 17.5 \mathrm{~nm}$ and $13.5 \mathrm{~nm}$.

of slope (or correlation length $a_{c}$ ) with increasing density. This is plausible, as the solgel process for the higher density tiles, i.e. a more concentrated initial TMos solution can be assumed to start off from more nucleation centers. In this case the number of skeletonforming "secondary" $\mathrm{SiO}_{2}$ particles (diameter a few $\mathrm{nm}$ ) /6/ is higher than for the lowdensity aerogels. This leads to density fluctuations for the skeleton which occur more frequently and are spacially less extended. We want to point out, that in addition to density also the $\mathrm{pH}$-value and, in general, the detailed recipe for gel formation and supercritical drying influence the transparence.

The scattered intensity increases by about 20 to $40 \%$ in our experiments if the scattering angle is changed from $\theta=160^{\circ}$ (backward scattering) to $\theta=20^{\circ}$ (forward scattering). This is typical for Rayleigh-Gans scattering $/ 7 /$ with inhomogeneities $a_{c} \approx \lambda / 20$. Our finding thus is in contrast to the strongly enhanced backscattering found in the displayed scattering intensity of reference /5/. A possible explanation for the unusual angular intensity profile in $/ 5 /$ could be intensity changes due to birefringence effects which were not taken into account, which however, are often observed in aerogel tiles. Another explanation could be the neglect of the correction term in equ. (5).

For comparison of the three geometries discussed in the above scattering measurements using the same aerogel sample were performed. As we can see from fig. 6 tile and cylinder geometry provide basicly the same angular scattering pattern. Expected deviations occur for the platelet geometry upon approaching the angle, when the angle of total reflection is approached, and for forward scattering. Obviously large surface inhomogeneities increase the scattering intensity in this angular region.

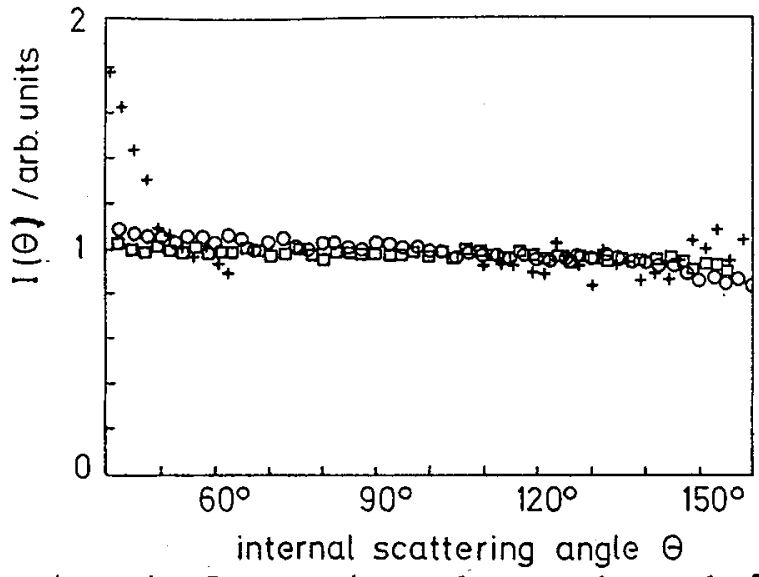

Fig. 6 - Scattering intensity I versus internal scattering angle $\theta$ for tile (D), cylinder ( 0 ) and platelet $(+)$ geometry.

We also investigated the sol-ge1 transition in a TEOS-ethano1-water solution /10/. Most evident is the strong forward scattering. resulting from impuriti'es. In the sol-gel process 
the scattering intensity increases over the total observed $Q$ range. Use of equ.(1) allows to extract a correlation length $a_{c} \approx 20 \mathrm{~nm}$, which remains constant for all times after gelation. We conclude that the spatial extend of the formed network has to remain fixed. only the density contrast increases after gelation (aging).

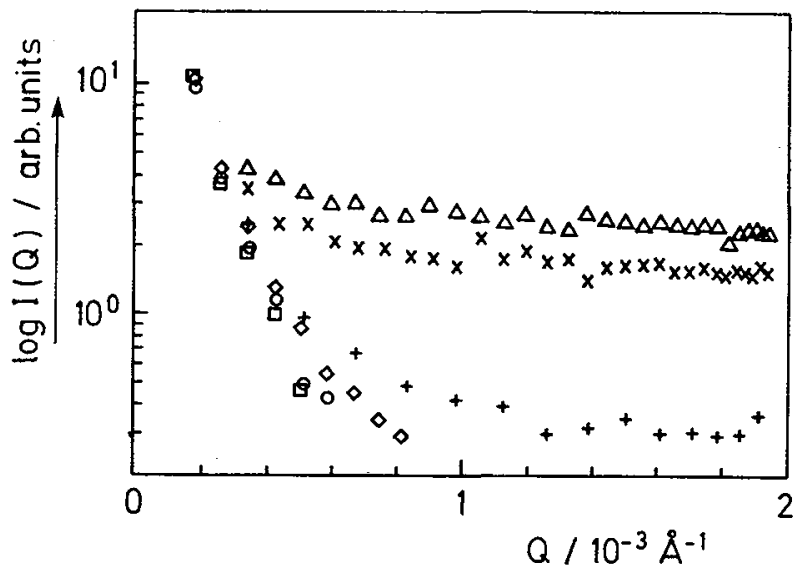

Fig. 7 - Change of scattered intensity $I$ of a gelling TEos-solution with time versus momentum transfer; time after mixing: $\square=0.5 \mathrm{~h}, 0=3.8 \mathrm{~h}, 0=6.6 \mathrm{~h},+=8.3 \mathrm{~h}, \mathrm{x}=3 \mathrm{~d}$ and $\Delta=6 \mathrm{~d}$.

\section{6 - oUTLOOK}

The constructed scattering apparatus is currently used to investigate granular aerogels as well as alcogels and gelling solutions. For these studies also a 320 nm He-Cd laser is employed. As the extinction mechanism is Rayleigh scattering a dramatic variation of $E$ with wavelength $\lambda$ is expected $: E \sim 1 / \lambda^{4}$. We want to point out that for these investigations with visible light a prerequisite is a size of the structural entities which is at least of the order of $\lambda / 60 \approx 10 \mathrm{~nm}$. This means for example that the growth of nm particles in a gelling solution cannot be detected with visible light (however with SAXS or SANS). However, the growth of large particles as in base-catalysed (ammonia concentration about 0.085 mol/1) sol-gel transitions is conveniently investigated with light scattering. The same holds for aging processes.

\section{REFERENCES}

11/ Fricke, J., Scientific American, May issue, (1988) 92

/2/ Fricke, J, Aerogels - a Fascinating Class of High - Performance Porous Solids, in : Aeroge1s, Springer Proc. in Physics 6, Ed. J. Fricke, Springer Verlag, Berlin, Heidelberg, New York, Tokyo, (1986) $\overline{2}$

13/ Schaefer, D.W., Martin, J.E., Hurd, A.J., and Keefer, K.D., Structure of Random Materials, in : Physics of Finely Divided Matter, Spring Proc. in Physics 5 , N. Boccara and M. Daoud (Eds), Springer Verlag Heidelberg (1985)

14/ Sinha, S.H., Freitoft, T., and Kjems, J., Observation of Power Law Correlations in Silica-Particle Aggregates by Sma11-Angle Neutron Scattering, in : Kinetics of Aggregation and Gelation, F: Family, D.P. Landau (Eds) E1sevier Sc. Pub1. London (1984)

15/ Hunt, A.J., and Berdah1, P., Mat. Res. Soc. 32 (1984) 275

16/ Fricke, J., and Reichenauer, G., J. Non Cryst. Solids $95 \& 96$ (1987) 1135

17) Kerker, M., The Scattering of Light, Academic Press, New York and London (1986)

$18 /$ Kistler, S.S., Nature 127 (1931) 741

19/ Poelz, G., Aerogels in Hign Energy Physics, in: Aeroge1s, Springer Proc. in Physics 6 , J. Fricke (Ed), Springer Verl. Berlin Heidelberg, New York Tokyo (1986) 176

110/ Mulder, C.A.M., and van Lierop, J.G., Preparation, Densification and Characterìzation of Autocalve Dried $\mathrm{SiO}_{2} \mathrm{Gels}$, in: Aerogels, Springer Proc. in Phys. 6, J. Fricke (Ed), Springer Verl. Berlin Heidelberg, New York Tokyo (1986) 68

\section{Acknowledgements}

We would like to thank Dr. G. Poelz/DESY, Hamburg, and Dr. S. Henning/Airglass, Lund for generous 1 y providing aerogel samples. 